\title{
Moments of Discounted Dividend Payments in the Sparre Andersen Model with a Constant Dividend Barrier*
}

\author{
Jiyang Tan ${ }^{1}$, Lin Xiao ${ }^{2}$, Shaoyue Liu ${ }^{1}$, Xiangqun Yang ${ }^{2}$ \\ ${ }^{1}$ Department of Statistics, Xiangtan University, Xiangtan, China \\ ${ }^{2}$ Department of Mathematics, Hunan Normal University, Changsha, China \\ E-mail: tanjiyang15@163.com \\ Received December 23, 2010; revised February 14, 2011; accepted February 26, 2011
}

\begin{abstract}
We consider the Sparre Andersen risk process in the presence of a constant dividend barrier, and propose a new expected discounted penalty function which is different from that of Gerber and Shiu. We find that iteration mothed can be used to compute the values of expected discounted dividends until ruin and the new penalty function. Applying the new function and the recursion method proposed in Section 5, we obtain the arbitrary moments of discounted dividend payments until ruin.
\end{abstract}

Keywords: Sparre Andersen Model, Expected Discounted Penalty Function, Constant Dividend Barrier, Recursion, Iteration

\section{Introduction}

The dividend problem in risk theory was brought out initially by De Finetti [1] and has been studied extensively in many literatures by now. Much of the literature on dividend theory is concentrated on the classical risk model, in which claims occur as a Poisson process. For the classical risk model with a barrier strategy, Lin et al. [2] studied the Gerber-Shiu discounted penalty function at ruin; Dickson and Waters [3] studied arbitrary moments of the discounted sum of dividend payments until ruin; Gerber et al. [4] recently developed methods for estimating the optimal dividend barrier.

The surplus process is not necessarily a compound Poisson process. Andersen [5] lets claims occur according to a more general renewal process. Since then, Sparre Andersen risk model was studied extensively. For some recent contributions to Sparre Andersen risk models with a dividend barrier, see [6-8]. It is worth mentioning that Albrecher et al. [8] studied a class of Sparre Andersen risk models with generalized Erlang(n) waiting times in the presence of a constant dividend barrier $b$, and gained some results on the distribution of dividend payments until ruin. It is natural to ask for developing some methods to get the distribution or moments of discounted

*Supported by the Natural Sciences Foundation of China (grant No. 10871064), and by Scientific Research Funds of Hunan Provincial Education Department (08C883), and Hunan Provincial Science and Technology Department (2009FJ3141). dividend payments in an arbitrary Sparre Andersen model.

In this paper, we consider the Sparre Andersen model with arbitrary distributed waiting times in the presence of a constant dividend barrier $b$. The analysis is focused on the evaluation of the new expected discounted penalty function defined in Section 2, which will permit us to obtain arbitrary moments of discounted dividend payments by applying the proposed recursion method.

\section{The Model}

Consider the Sparre Andersen risk model, which is given by

$$
U(t)=u+c t-S(t)=u+c t-\sum_{i=1}^{N(t)} X_{i}
$$

where $u \geq 0$ is the initial surplus, $c$ is a constant premium rate, $S(t)$ is the aggregate claim up to time $t, N(t)$ is the number of claims occurring in $(0, t]$, and $X_{i}$ is the $i$ th claim. Let $M_{1}, M_{2}, \cdots$ denote the inter-claim times, and assume that

$$
L_{n}=\sum_{k=1}^{n} M_{k} .
$$

We assume that $\left\{X_{n}, n \geq 1\right\}$ and $\left\{M_{n}, n \geq 1\right\}$ are independent sequences of i.i.d. non-negative random variables. $\left\{X_{n}, n \geq 1\right\}$ have a common distribution 
$F(x)=\operatorname{Pr}\left\{X_{1} \leq x\right\} \quad$ with $F(0)=0$, and $\left\{M_{n}, n \geq 1\right\}$ have a common distribution $G(x)=\operatorname{Pr}\left\{M_{1} \leq x\right\}$ with $G(0)=0$. Assume $E M_{1}<\infty$.

The risk process (2.1) is now modified by introducing a constant dividend barrier $b(b \geq 0)$, i.e. whenever the surplus process reaches the level $b$, the premium income is paid out as dividends to shareholders and the modified surplus process remains at level $b$ until the occurrence of the next claim. Let $\left\{U_{b}(t)\right\}$ denote the modified surplus process, and the random variable $D(u, b)$ denote the sum of the discounted dividend payments until ruin (with force of interest $\delta_{0} \geq 0$ ). In the sequel we will be interested in the $k$ th moment of the sum of discounted dividend payments

$$
W_{b}^{(k)}(u)=E\left[D^{k}(u, b) \mid U(0)=u\right], \quad k=1,2, \cdots .
$$

Let $W_{b}^{(1)}(u)=W_{b}(u)$, which is the expectation. We will always assume that $0 \leq u \leq b$.

Let the time of ruin for this modified surplus process $\left\{U_{b}(t)\right\}$ be

$$
T=T_{b}(u)=\inf \left\{t \geq 0: U_{b}(t)<0\right\}(\infty \text { otherwise })
$$

Obviously, $T$ must be some $L_{i}$. Define the stochastic time $\tau$ by

$$
\tau=\tau_{b}(u)=\inf \left\{L_{i}: U\left(L_{i}-\right)>b, i \geq 1\right\} .
$$

For $x \geq 0$, let $v(x)$ be a non-negative measurable function. For $\delta_{0} \geq 0$ we define a new expected discounted penalty function by

$$
\varphi_{b}(u)=E\left[e^{-\delta \tau} v(U(\tau-)) I(\tau \leq T) \mid U(0)=u\right], 0 \leq u \leq b
$$

where $I(\cdot)$ is the indicator function. The function $\varphi_{b}(u)$ is similar to (but different from) Gerber-Shiu expected discounted penalty function.

Let $S$ denote the space of real-valued measurable functions on $[0, b]$. Choosing the metric defined by

$$
\mathrm{d}(x, y)=\sup _{u \in[0, b]}|x(u)-y(u)|, \forall x, y \in S,
$$

$S=(S, d)$ is complete metric space. Obviously, $W_{b}(u)$ and $\varphi_{b}(u)$ are contained within $S$ because of the measurable property of $v(x)$ and the monotone property of $W_{b}(u)$.

\section{The Expectation of Discounted Dividends Until Ruin}

Define an operator $\hat{T}: S \rightarrow S$ by

$$
\begin{aligned}
\hat{T} f= & \int_{0}^{(b-u) / c} \int_{0}^{u+c t} f(u+c t-x) e^{-\delta_{0} t} \mathrm{~d} F(x) \mathrm{d} G(t) \\
& +\int_{(b-u) / c}^{\infty} \int_{0}^{b} f(b-x) e^{-\delta_{0} t} \mathrm{~d} F(x) \mathrm{d} G(t)+D_{1},
\end{aligned}
$$

where $f=f(u)$ is an arbitrary function in $S$, and
$D_{1}=$

$\begin{cases}\frac{c}{\delta_{0}} e^{\frac{\delta_{0}}{c}(u-b)}\left[1-G\left(\frac{b-u}{c}\right)\right]-\frac{c}{\delta_{0}} \int_{(b-u) / c}^{\infty} e^{-\delta_{0} t} \mathrm{~d} G(t), & \delta_{0}>0 \\ (u-b)\left[1-G\left(\frac{b-u}{c}\right)\right]+c \int_{(b-u) / c}^{\infty} t \mathrm{~d} G(t), & \delta_{0}=0\end{cases}$

Theorem 1. Under the assumption that $F(b)<1$ or $\delta_{0}>0$, the function $W_{b}(u)$ is equal to

$$
W_{b}(u)=\left(\lim _{n \rightarrow \infty} \hat{T}^{n} f\right)(u), \quad \forall b \geq 0 .
$$

As an approximation of $W_{b}(u),\left(\hat{T}^{n} f\right)(u)$ satisfies $\sup _{u \in[0, b]}\left|\left(\hat{T}^{n} f\right)(u)-W_{b}(u)\right| \leq \frac{\alpha^{n}}{1-\alpha} \sup _{u \in[0, b]}|f(u)-(\hat{T} f)(u)|$,

where $\alpha=F(b) \int_{0}^{\infty} e^{-\delta_{0} t} \mathrm{~d} G(t)$.

Proof. Ruin can not occur in $\left(0, L_{1}\right)$ and the expectation of the discounted dividends paid out in this period is $D_{1}$. By the renewal argument we have

$$
W_{b}(u)=E\left[e^{-\delta_{0} L_{1}} W_{b}\left(\left(u+c L_{1}\right) \wedge b-X_{1}\right)\right]+D_{1},
$$

namely,

$$
\begin{aligned}
W_{b}(u)= & \int_{0}^{(b-u) / c} \int_{0}^{u+c t} W_{b}(u+c t-x) e^{-\delta_{0} t} \mathrm{~d} F(x) \mathrm{d} G(t) \\
& +\int_{(b-u) / c}^{\infty} \int_{0}^{b} W_{b}(b-x) e^{-\delta_{0} t} d F(x) \mathrm{d} G(t)+D_{1},
\end{aligned}
$$

which is an integral equation for $W_{b}(u)$. Since $W_{b}(u) \in S$, the Equation (3.5) can be rewritten as

$$
W_{b}(u)=\left(\hat{T} W_{b}\right)(u) \text {. }
$$

For arbitrary $f, g \in S$, we have

$$
\mathrm{d}(\hat{T} f, \hat{T} g) \leq \sup _{u \in[0, b]}
$$

$$
\begin{aligned}
& \left\{\int_{0}^{(b-u) / c} \int_{0}^{u+c t}|f(u+c t-x)-g(u+c t-x)| e^{-\delta_{0} t} \mathrm{~d} F(x) \mathrm{d} G(t)\right. \\
& \left.\quad+\int_{(b-u) / c}^{\infty} \int_{0}^{b}|f(b-x)-g(b-x)| e^{-\delta_{0} t} \mathrm{~d} F(x) \mathrm{d} G(t)\right\} \\
& \leq \sup _{u \in[0, b]}|f(u)-g(u)| \cdot \sup _{u \in[0, b]}\left\{\int_{0}^{(b-u) / c} \int_{0}^{u+c t} e^{-\delta_{0} t} \mathrm{~d} F(x) \mathrm{d} G(t)\right. \\
& \left.\quad+\int_{(b-u) / c}^{\infty} \int_{0}^{b} e^{-\delta_{0} t} \mathrm{~d} F(x) \mathrm{d} G(t)\right\} \\
& \leq \mathrm{d}(f, g) \cdot \int_{0}^{\infty} \int_{0}^{b} e^{-\delta_{0} t} \mathrm{~d} F(x) \mathrm{d} G(t)
\end{aligned}
$$

which leads to

$$
d(\hat{T} f, \hat{T} g) \leq d(f, g) F(b) \int_{0}^{\infty} e^{-\delta_{0} t} \mathrm{~d} G(t) .
$$


Thus, $\hat{T}$ is a contraction on $S$ if $F(b)<1$ or $\delta_{0}>0$ (see [9]). (3.3) and (3.4) follow.

Note that when $b=0$ we have, for $\delta_{0} \geq 0$,

$$
W_{0}(0)=\frac{c}{\delta_{0}}\left\{1-\int_{0}^{\infty} e^{-\delta_{0} t} \mathrm{~d} G(t)\right\},
$$

and for $\delta_{0}=0, W_{0}(0)=c E\left[M_{1}\right]$.

Theorem 1 gives an iteration procedure by which we can obtain approximations to $W_{b}(u)$ and error bounds. In order to gain more information about the sum of discounted dividend payments until ruin, we discuss the new expected discounted penalty function in Section 4.

\section{Expected Discounted Penalty Function}

Define an integral operator as follows:

$$
\begin{aligned}
\widetilde{T} f= & \int_{0}^{(b-u) / c} \int_{0}^{u+c t} f(u+c t-x) e^{-\delta t} \mathrm{~d} F(x) \mathrm{d} G(t) \\
& +\int_{(b-u) / c}^{\infty} e^{-\delta t} v(u+c t) \mathrm{d} G(t), \forall f \in S .
\end{aligned}
$$

Theorem 2. The penalty function $\varphi_{b}(u)$ is the solution of the integral equation

$$
\varphi_{b}(u)=\left(\widetilde{T} \varphi_{b}\right)(u) .
$$

And under the assumption that $F(b)<1$ or $G(b / c)<1$ or $\delta>0$, the penalty function $\varphi_{b}(u)$ is the unique solution and equal to

$$
\varphi_{b}(u)=\left(\lim _{n \rightarrow \infty} \widetilde{T}^{n} f\right)(u),
$$

where $f$ is an arbitrary real-valued measurable function.

Proof. The discrete time process $\left\{U_{b}\left(L_{n}\right), n=0,1, \cdots\right\}$ has stationary and independent increments. By the renewal argument, we have the integral Equation (4.2). The uniqueness and the result (4.3) are due to the fact that $\widetilde{T}$ is a contraction under the conditions $F(b)<1$ or $\mathrm{G}(b / c)<1$ or $\delta>0$. In fact, for arbitrary real-valued measurable functions $y(u)$ and $z(u)$ on $[0, b]$, we have

$$
\begin{aligned}
& \sup _{u \in[0, b]}|\widetilde{T} y-\widetilde{T} z| \leq \sup _{u \in[0, b]} \\
& \left\{\int_{0}^{(b-u) / c} \int_{0}^{u+c t}|y(u+c t-x)-z(u+c t-x)| e^{-\delta t} \mathrm{~d} F(x) \mathrm{d} G(t)\right\} \\
& \leq \sup _{u \in[0, b]}|y(u)-z(u)| \cdot \sup _{u \in[0, b]} \int_{0}^{(b-u) / c} e^{-\delta t} F(u+c t) \mathrm{d} G(t) \\
& \leq \sup _{u \in[0, b]}|y(u)-z(u)| \cdot F(b) \int_{0}^{b / c} e^{-\delta t} \mathrm{~d} G(t),
\end{aligned}
$$

where $F(b) \int_{0}^{b / c} e^{-\delta t} \mathrm{~d} G(t)<1$. The results are proven.

Remark. 1) Obviously, when $u=b$ we can obtain the explicit expression

$$
\varphi_{b}(b)=\int_{0}^{\infty} e^{-\delta t} v(b+c t) \mathrm{d} G(t)
$$

2) According to Theorem 2, we can obtain the approximation of $\varphi_{b}(u)$ by the iteration method. As an approximation of $\varphi_{b}(u), \quad \widetilde{T}^{n} f$ satisfy

$$
\sup _{u \in[0, b]}\left|\left(\widetilde{T}^{n} f\right)(u)-\varphi_{b}(u)\right| \leq \frac{\alpha^{n}}{1-\alpha} \sup _{u \in[0, b]}|f(u)-(\widetilde{T} f)(u)|,
$$

where $\quad \alpha=\sup _{u \in[0, b]} \int_{0}^{(b-u) / c} e^{-\delta t} F(u+c t) \mathrm{d} G(t)$. The error bound (4.5) can be used for estimating the number of steps necessary to reach a given accuracy.

Now, we give some examples of dividend-related quantities (such as the probability of the event $\tau_{b}(u) \leq T_{b}(u)$, the $k$ th moment of the discounted dividends paid out in time period $(0, \tau)$, and the distribution function of the sum of the dividends paid out in time period $(0, \tau)$, etc.) to illustrate applications of Theorem 2 .

Example 4.1. Letting $v(x)=1$ and $\delta=0$, we have

$$
\varphi_{b}(u)=\operatorname{Pr}\left(\tau_{b}(u) \leq T_{b}(u)\right) .
$$

The contraction $\widetilde{T}$ is defined by

$$
\begin{aligned}
(\widetilde{T} f)(u)= & \int_{0}^{(b-u) / c} \int_{0}^{u+c t} f(u+c t-x) \mathrm{d} F(x) \mathrm{d} G(t) \\
& +1-G\left(\frac{b-u}{c}\right), \quad \forall f \in S .
\end{aligned}
$$

For any real-valued measurable function $f$ on $[0, b]$, we have

$$
\operatorname{Pr}\left(\tau_{b}(u) \leq T_{b}(u)\right)=\lim _{n \rightarrow \infty} \tilde{T}^{n} f .
$$

From (4.4), it is easily seen that

$$
\operatorname{Pr}\left(\tau_{b}(b) \leq T_{b}(b)\right)=1 .
$$

Example 4.2. Letting $v(x)=1$ and $\delta \geq 0$, we have

$$
\varphi_{b}(u)=E\left[e^{-\delta \tau} I\left(\tau_{b}(u) \leq T_{b}(u)\right)\right] .
$$

Let $R_{\delta}(u)$ denote this function. Note that

$$
R_{0}(u)=\operatorname{Pr}\left(\tau_{b}(u) \leq T_{b}(u)\right) .
$$

Here, the contraction $\widetilde{T}$ is defined by

$$
\begin{aligned}
(\widetilde{T} f)(u)= & \int_{0}^{(b-u) / c} \int_{0}^{u+c t} e^{-\delta t} f(u+c t-x) \mathrm{d} F(x) \mathrm{d} G(t) \\
& +\int_{(b-u) / c}^{\infty} e^{-\delta t} \mathrm{~d} G(t) .
\end{aligned}
$$

Choosing $f=0$, we have

$$
R_{\delta}(u)=\lim _{n \rightarrow \infty} \widetilde{T}^{n}\left[\int_{(b-u) / c}^{\infty} e^{-\delta t} \mathrm{~d} G(t)\right] .
$$

The error estimate is 


$$
\sup _{u \in[0, b]}\left|\left(\widetilde{T}^{n} f\right)(u)-R_{\delta}(u)\right| \leq \frac{\alpha^{n}}{1-\alpha} \int_{0}^{\infty} e^{-\delta t} \mathrm{~d} G(t) .
$$

By (4.4), we have

$$
R_{\delta}(b)=\int_{0}^{\infty} e^{-\delta t} \mathrm{~d} G(t)
$$

Example 4.3. The insurer will continuously pay dividends in time period $(\tau-(U(\tau-)-b) / c, \tau)$ at rate $c$ when $\tau \leq T$. Set

$$
w(x)=\frac{c}{\delta_{0}}\left[e^{\frac{\delta_{0}}{c}(x-b)}-1\right] .
$$

Then, the present value of these dividends is $e^{-\delta_{0} \tau} w(U(\tau-))$. For arbitrary $i=0,1, \cdots$, letting $v(x)=w^{i}(x)$ and $\delta \geq 0$, yields

$$
\varphi_{b}(u)=E\left[e^{-\delta \tau} w^{i}(U(\tau-)) I\left(\tau_{b}(u) \leq T_{b}(u)\right)\right],
$$

which we denote by $A_{\delta, i}(u)$. The contraction $\widetilde{T}$ is defined by

$$
\begin{aligned}
(\tilde{T} f)(u)= & \int_{0}^{(b-u) / c} \int_{0}^{u+c t} e^{-\delta t} f(u+c t-x) \mathrm{d} F(x) \mathrm{d} G(t) \\
& +\int_{(b-u) / c}^{\infty} e^{-\delta t} w^{i}(u+c t) \mathrm{d} G(t) .
\end{aligned}
$$

Choosing $f(u)=0$, we have

$$
A_{\delta, i}(u)=\lim _{n \rightarrow \infty} \widetilde{T}^{n}\left[\int_{(b-u) / c}^{\infty} e^{-\delta t} w^{i}(u+c t) \mathrm{d} G(t)\right] .
$$

The error estimate is

$$
\begin{aligned}
& \sup _{u \in[0, b]}\left|\left(\widetilde{T}^{n} f\right)(u)-A_{\delta, i}(u)\right| \\
& \leq \frac{c^{i} \alpha^{n}}{\delta_{0}^{i}(1-\alpha)} \int_{0}^{\infty} e^{-\delta t}\left[e^{\delta_{0} t}-1\right]^{i} \mathrm{~d} G(t) .
\end{aligned}
$$

Note that if $\delta=i \delta_{0}$, then $A_{\delta, i}(u)=A_{i \delta_{0}, i}(u)$ is the $i$ th moment of the discounted dividends paid out in the period $(0, \tau)$; if $i=0$, i.e. $v(x)=w^{0}(x)$, then $A_{\delta, 0}(u)=R_{\delta}(u)$. In addition, it should be pointed out that

$$
A_{\delta, i}(b)=\int_{0}^{\infty} e^{-\delta t} w^{i}(u+c t) \mathrm{d} G(t)
$$

Example 4.4. Letting $\delta=0$, and $v(x)=I(x-b \leq z)$ for arbitrary $z \geq 0$, we have

$$
\varphi_{b}(u)=\operatorname{Pr}[U(\tau-)-b \leq z, \tau \leq T],
$$

which is the distribution function of the cumulative dividends in time period $(\tau-(U(\tau-)-b) / c, \tau)$. We denote this distribution function by $\Phi(u, z)$. The contraction $\widetilde{T}$ is defined by

$$
\begin{aligned}
(\widetilde{T} f)(u)= & \int_{0}^{(b-u) / c} \int_{0}^{u+c t} f(u+c t-x) \mathrm{d} F(x) \mathrm{d} G(t) \\
& +G\left(\frac{b-u+z}{c}\right)-G\left(\frac{b-u}{c}\right) .
\end{aligned}
$$

Choosing $f(u)=0$, we have

$$
\Phi(u, z)=\lim _{n \rightarrow \infty} \widetilde{T}^{n}\left[G\left(\frac{b-u+z}{c}\right)-G\left(\frac{b-u}{c}\right)\right] .
$$

The error estimate is

$$
\sup _{u \in[0, b]}\left|\left(\widetilde{T}^{n} f\right)(u)-\Phi(u, z)\right| \leq \frac{\alpha^{n}}{1-\alpha} G\left(\frac{b+z}{c}\right),
$$

where

$$
\alpha=\sup _{u \in[0, b]} \int_{0}^{(b-u) / c} F(u+c t) d G(t)
$$

\section{The kth Moment of Discounted Dividend Payments Until Ruin}

In this section, we use $\tau_{1}(u)$ (or $\tau_{1}$ ) instead of $\tau_{b}(u)$ and use $T_{1}(u)$ (or $T_{1}$ ) instead of $T_{b}(u)$.

If ruin doesn't occur at time $\tau_{1}(u)$ and $U_{b}\left(\tau_{1}\right)=u_{2}$ $\left(0 \leq u_{2}<b\right)$, we view the process as "starting again" with "initial surplus" $u_{2}$, and similarly to the definitions of $T_{b}(u)$ and $\tau_{b}(u)$, define the stochastic times $T_{2}=T_{2}\left(u_{2}\right)$ and $\tau_{2}=\tau_{2}\left(u_{2}\right)$ respectively for the new pro cess $U_{b}\left(u_{2}\right)$. If ruin doesn't occur at $\tau_{2}$ either, similarly we define $T_{3}=T_{3}\left(u_{3}\right)$ and $\tau_{3}=\tau_{3}\left(u_{3}\right)$. Applying repeatedly the idea of "starting again", we can define two sequences of mutually independent random variables $\left\{T_{i}, i \geq 1\right\}$ and $\left\{\tau_{i}, i \geq 1\right\}$. Suppose that the claim amount at $\tau_{i}$ is $X_{(i+1)}$. Then, $u_{i}=b-X_{(i)}$ ( $i \geq 2)$.

Further, suppose that

$$
\begin{gathered}
\xi_{1}=e^{-\delta_{0} \tau_{1}} I\left(\tau_{1} \leq T_{1}\right), \\
\xi_{i}=I\left(X_{(i)} \leq b\right) \cdot e^{-\delta_{0} \tau_{i}} I\left(\tau_{i} \leq T_{i}\right), \quad i \geq 2 .
\end{gathered}
$$

Then, $\xi_{i}(i \geq 2)$ are i.i.d. random variables and independent of $\xi_{1}$. According to Example 2 in Section 4, we have $E \xi_{1}^{k}=R_{k \delta_{0}}(u)$ and

$$
\begin{aligned}
E \xi_{2}^{k} & =E\left[I\left(X_{(2)} \leq b\right) R_{k \delta_{0}}\left(b-X_{(2)}\right)\right] \\
& =\int_{0}^{b} R_{k \delta_{0}}(b-x) \mathrm{d} F(x) .
\end{aligned}
$$

We denote $E \xi_{2}^{k}$ by $\bar{R}_{k}$. Assume that

$$
\begin{gathered}
\eta_{1}=e^{-\delta_{0} \tau_{1}} w\left(U\left(\tau_{1}-\right)\right) I\left(\tau_{1} \leq T_{1}\right), \\
\eta_{i}=I\left(X_{(i)} \leq b\right) \cdot e^{-\delta_{0} \tau_{i}} w\left(U\left(\tau_{i}-\right)\right) I\left(\tau_{i} \leq T_{i}\right), \quad i \geq 2,
\end{gathered}
$$

which is the "present value" of the dividends paid in time period $\left(0, \tau_{i}\right)$ in the $(i-1)$ th "starting again" process. Obviously, $\eta_{i}(i \geq 2)$ are i.i.d. random variables and random vectors $\left(\xi_{1}, \eta_{1}\right),\left(\xi_{2}, \eta_{2}\right), \cdots$ are mutually independent. According to Example 3 in Section 4, we have 


$$
\begin{gathered}
E\left(\xi_{1}^{i} \eta_{1}^{j}\right)=A_{(i+j) \delta_{0}, j}(u), \quad i, j \geq 0, \\
E\left(\xi_{2}^{i} \eta_{2}^{j}\right)=E\left(I\left(X_{(2)} \leq b\right) A_{(i+j) \delta_{0}, j}\left(b-X_{(2)}\right)\right) \\
=\int_{0}^{b} A_{(i+j) \delta_{0}, j}(b-x) \mathrm{d} F(x)(i, j \geq 0) .
\end{gathered}
$$

We denote $E\left(\xi_{2}^{i} \eta_{2}^{j}\right)$ by $\bar{A}_{i+j, j}$.

Theorem 3. The $k$ th moment of the sum of discounted dividend payments until ruin is equal to

$$
W_{b}^{(k)}(u)=\sum_{i=0}^{k} \frac{k !}{i !(k-i) !} A_{k \delta_{0}, i}(u) x_{k-i}, k=1.2, \cdots,
$$

where $x_{j}(j=0,1,2, \cdots)$ satisfy the following recursive formulas:

$$
x_{0}=1 ; \quad x_{j}=\sum_{i=1}^{j} \frac{j !}{i !(j-i) !} \bar{A}_{j, i} x_{j-i} /\left(1-\bar{R}_{j}\right), \forall j>0 .
$$

Proof. It is easily seen that the sum of the discounted dividend payments until ruin is equal to

$$
D(u, b)=\eta_{1}+\xi_{1} \eta_{2}+\xi_{1} \xi_{2} \eta_{3}+\cdots=\sum_{i=1}^{\infty}\left(\eta_{i} \prod_{k=1}^{i-1} \xi_{k}\right),
$$

where we adopt the convention that $\prod_{k=1}^{0} \xi_{k}=1$. Thus, we have

$$
\begin{aligned}
D^{k}(u, b) & =\sum_{i=0}^{k} \frac{k !}{i !(k-i) !} \eta_{1}^{i}\left(\xi_{1} \eta_{2}+\xi_{1} \xi_{2} \eta_{3}+\xi_{1} \xi_{2} \xi_{3} \eta_{4}+\cdots\right)^{k-i} \\
& =\sum_{i=0}^{k} \frac{k !}{i !(k-i) !} \eta_{1}^{i} \xi_{1}^{k-i}\left(\eta_{2}+\xi_{2} \eta_{3}+\xi_{2} \xi_{3} \eta_{4}+\cdots\right)^{k-i} .
\end{aligned}
$$

Taking expectation of (5.7) yields

$$
W_{b}^{(k)}(u)=\sum_{i=0}^{k} \frac{k !}{i !(k-i) !} A_{k \delta_{0}, i}(u) E\left[\eta_{2}+\xi_{2} \eta_{3}+\xi_{2} \xi_{3} \eta_{4}+\cdots\right]^{k-i},
$$$$
k=1,2, \cdots \text {. }
$$

Note that

$$
\begin{aligned}
& E\left[\eta_{2}+\xi_{2} \eta_{3}+\xi_{2} \xi_{3} \eta_{4}+\cdots\right]^{j} \\
& =E \sum_{i=0}^{j} \frac{j !}{i !(j-i) !} \eta_{2}^{i}\left(\xi_{2} \eta_{3}+\xi_{2} \xi_{3} \eta_{4}+\xi_{2} \xi_{3} \xi_{4} \eta_{5}+\cdots\right)^{j-i} \\
& =E \sum_{i=0}^{j} \frac{j !}{i !(j-i) !^{j-i} \eta_{2}^{i}\left(\eta_{3}+\xi_{3} \eta_{4}+\xi_{3} \xi_{4} \eta_{5}+\cdots\right)^{j-i}} \\
& =\sum_{i=0}^{j} \frac{j !}{i !(j-i) !} E\left[\xi_{2}^{j-i} \eta_{2}^{i}\right] \cdot E\left(\eta_{3}+\xi_{3} \eta_{4}+\xi_{3} \xi_{4} \eta_{5}+\cdots\right)^{j-i} \\
& =\sum_{i=0}^{j} \frac{j !}{i !(j-i) !} \bar{A}_{j, i} \cdot E\left(\eta_{2}+\xi_{2} \eta_{3}+\xi_{2} \xi_{3} \eta_{4}+\cdots\right)^{j-i} .
\end{aligned}
$$

Since $\bar{A}_{j, 0}=\bar{R}_{j}$, it follows that, for $j=1,2, \cdots$,

$$
\begin{aligned}
& E\left(\eta_{2}+\xi_{2} \eta_{3}+\xi_{2} \xi_{3} \eta_{4}+\cdots\right)^{j} \\
& =\sum_{i=1}^{j} \frac{j !}{i !(j-i) !} \bar{A}_{j, i} \frac{E\left(\eta_{2}+\xi_{2} \eta_{3}+\xi_{2} \xi_{3} \eta_{4}+\cdots\right)^{j-i}}{1-\bar{R}_{j}},
\end{aligned}
$$

which leads to

$$
x_{j}=E\left[\eta_{2}+\xi_{2} \eta_{3}+\xi_{2} \xi_{3} \eta_{4}+\cdots\right]^{j}, \quad j=0,1,2, \cdots .
$$

From (5.8) and (5.10), we get (5.4).

\section{Numerical Illustration}

As an illustration of the results in Sections 3 and 5, consider the case of a Sparre Andersen model with Erlang (2) interclaim times and Erlang (2) claim amounts, i.e.

$$
G(t)=F(t)=1-(\lambda t+1) e^{-\lambda t} \quad(t \geq 0) .
$$

Let $\lambda=2, c=1.1$, and $\delta_{0}=0.03$. These accord with the assumptions in the Example 4.1 in [8].

Let us first consider the expectation of discounted dividend payments until ruin. Given $b=0,1,2, \cdots, 10$ respectively, according to Theorem 1 we choose the function $f(u)=0$ and determine a number of steps $n$ necessary to obtain $\left(\hat{T}^{n} f\right)(u)$ as an approximation for $W_{b}(u)$ such that

$$
\sup _{u \in[0, b]}\left|\left(\hat{T}^{n} f\right)(u)-W_{b}(u)\right|<0.0001,
$$

see Table 1. Using the iteration procedure, we get some approximate values of $W_{b}(u)$ in Table 2. Comparing with the exact values given by Albrecher et al. [8], it can be seen that the approximate values in Table $\mathbf{2}$ are fairly good. Note that, when $b=0$, the numerical value 1.076 is obtained by (3.8).

For the $k$ th moment of discounted dividend payments until ruin, we need compute $A_{j \delta_{0}, i}(u), \bar{A}_{j, i} \quad(j=1,2$, $\cdots, k ; i=1,2, \cdots, j)$ and $\bar{R}_{j}(j \stackrel{A}{=} 1,2, \cdots, k)$. In this example, we only consider three cases: $k=1,2,3$. In order to reach an accuracy of 0.00001 , the necessary numbers of steps for iteration are given in Table 3. By formula (5.4), we obtain the approximate values for $W_{b}(u)$ again, see Table 4. In Table 5, the approximate values for the standard deviation $\sqrt{W_{b}^{(2)}(u)-W_{b}^{2}(u)}$ are given. Comparing with the Table 2 in [8], one can find the approximate values are very excellent too. In Table 6, approximations for the third moment are displayed. In addition, we point out that when $b=0$ the number of steps is not offered in Table 3, and the corresponding approximations in Tables 4-6 can be obtained by (4.8) (4.12) and (4.16).

\section{Summary}

As shown in Section 6, the iteration method and the re- 
Table 1. Numbers of steps for computing the expectation $W_{b}(u)$ by Theorem 1 .

\begin{tabular}{cccccccccccc}
\hline$b$ & 1 & 2 & 3 & 4 & 5 & 6 & 7 & 9 & 10 \\
\hline$n$ & 19 & 76 & 197 & 283 & 307 & 311 & 312 & 312 & 312 \\
\hline
\end{tabular}

Table 2. Approximations for the expectation $W_{b}(u)$ by Theorem 1.

\begin{tabular}{|c|c|c|c|c|c|c|c|c|c|c|c|}
\hline$b^{u}$ & 0 & 1 & 2 & 3 & 4 & 5 & 6 & 7 & 8 & 9 & 10 \\
\hline 0 & $1.076 \backslash 1.076$ & & & & & & & & & & \\
\hline 1 & $0.836 \backslash 0.836$ & $1.808 \backslash_{1.808}$ & & & & & & & & & \\
\hline 2 & $0.856 \backslash 0.856$ & $1.847 \backslash_{1.847}$ & $2.846 \mid 2.846$ & & & & & & & & \\
\hline 3 & $0.848 \backslash 0.848$ & $1.829 \backslash_{1.828}$ & $2.815 \backslash 2.815$ & $3.803 \backslash_{3.803}$ & & & & & & & \\
\hline 4 & $0.802 \backslash 0.801$ & $1.728 \backslash_{1.728}$ & $2.661 \mid 2.661$ & $3.597 \backslash_{3.597}$ & $4.574 \backslash_{4.574}$ & $5.143 \backslash 5.143$ & & & & & \\
\hline 5 & $0.730 \backslash 0.730$ & $1.575 \backslash_{1.575}$ & $2.424 \mid 2.424$ & $3.277 \backslash_{3.277}$ & $4.175 \backslash 4.174$ & $4.575 \backslash 4.575$ & & & & & \\
\hline 6 & $0.648 \backslash_{0.648}$ & $1.397 \backslash_{1.397}$ & $2.151 \mid 2.151$ & $2.908 \backslash 2.908$ & $3.705 \backslash 3.705$ & $3.988 \backslash 3.988$ & $5.538 \backslash 5.538$ & & & & \\
\hline 7 & $0.565 \backslash 0.565$ & $1.218 \backslash_{1.218}$ & $1.875 \backslash_{1.875}$ & $2.535 \backslash 2.535$ & $3.229 \backslash 3.229$ & $\left.3.436\right|_{3.436}$ & $4.840 \backslash 4.840$ & $5.799 \backslash 5.799$ & & & \\
\hline 8 & $0.487 \backslash 0.486$ & $1.049 \backslash_{1.049}$ & $1.615 \backslash_{1.615}$ & $\left.2.184\right|_{2.184}$ & $2.782 \backslash 2.782$ & $2.938 \mid 2.9$ & $4.170 \backslash 4.170$ & $5.010 \backslash 5.010$ & $5.967 \backslash 5.967$ & & \\
\hline 9 & $0.416 \backslash 0.416$ & $0.897 \backslash 0.897$ & $1.381 \backslash 1.381$ & $1.867 \backslash_{1.867}$ & $2.379 \backslash 2.379$ & 38 & $3.566 \backslash 3.566$ & $4.285 \backslash 4.285$ & $5.118 \backslash 5.118$ & $6.073 \backslash_{6.073}$ & \\
\hline 10 & 0.354 & 0.763 & 1.175 & 1.589 & 2.025 & 2.500 & 3.035 & 3.647 & 4.357 & 5.185 & 6.139 \\
\hline
\end{tabular}

a. The exact values given by Albrecher et al. (2005) are in smaller size after $\backslash$.

Table 3. Numbers of steps for computing $A_{\delta, i}(u) \quad(i=0,1,2,3)$.

\begin{tabular}{cccccccccccc}
\hline$\delta \backslash n \backslash u$ & 1 & 2 & 3 & 4 & 5 & 6 & 7 & 8 & 9 & 10 \\
\hline 0.03 & 8 & 18 & 39 & 78 & 141 & 220 & 292 & 340 & 366 & 379 \\
0.06 & 8 & 18 & 36 & 67 & 106 & 143 & 169 & 184 & 191 & 194 \\
0.09 & 8 & 17 & 34 & 58 & 85 & 107 & 120 & 127 & 130 & 131 \\
\hline
\end{tabular}

Table 4. Approximations for the expectation $W_{b}(u)$ by Formula (5.4).

\begin{tabular}{|c|c|c|c|c|c|c|c|c|c|c|c|}
\hline$b^{u}$ & 0 & 1 & 2 & 3 & 4 & 5 & 6 & 7 & 8 & 9 & 10 \\
\hline 0 & 1.0757 & & & & & & & & & & \\
\hline 1 & 0.8357 & 1.8082 & & & & & & & & & \\
\hline 2 & 0.8564 & 1.8469 & 2.8462 & & & & & & & & \\
\hline 3 & 0.8480 & 1.8285 & 2.8146 & 3.8027 & & & & & & & \\
\hline 4 & 0.8015 & 1.7283 & 2.6605 & 3.5969 & 4.5740 & & & & & & \\
\hline 5 & 0.7302 & 1.5745 & 2.4239 & 3.2774 & 4.1745 & 5.1433 & & & & & \\
\hline 6 & 0.6479 & 1.3971 & 2.1507 & 2.9081 & 3.7048 & 4.5745 & 5.5376 & & & & \\
\hline 7 & 0.5647 & 1.2177 & 1.8746 & 2.5347 & 3.2292 & 3.9881 & 4.8396 & 5.7989 & & & \\
\hline 8 & 0.4865 & 1.0490 & 1.6149 & 2.1836 & 2.7819 & 3.4357 & 4.1703 & 5.0101 & 5.9670 & & \\
\hline 9 & 0.4160 & 0.8970 & 1.3809 & 1.8672 & 2.3788 & 2.9379 & 3.5661 & 4.2853 & 5.1178 & 6.0731 & \\
\hline 10 & 0.3541 & 0.7635 & 1.1753 & 1.5893 & 2.0246 & 2.5005 & 3.0352 & 3.6474 & 4.3570 & 5.1849 & 6.1393 \\
\hline
\end{tabular}

cursion method proposed in this paper give good approximations for the arbitrary moments of discounted dividend payments until ruin. The exact mothed presented by
Albrecher et al. [8] can only be used in the model with generalied Erlang $(n)$-distributed inter-claim times. The purpose of this paper is to find an approach which can be 
Table 5. Approximations for the standard deviation $\sqrt{W_{b}^{(2)}(u)-W_{b}^{2}(u)}$.

\begin{tabular}{|c|c|c|c|c|c|c|c|c|c|c|}
\hline$b^{u}$ & 0 & 1 & 2 & 3 & 4 & 5 & 6 & 7 & 8 & 9 \\
\hline 0 & $0.7440 \backslash 0.744$ & & & & & & & & & \\
\hline 1 & $1.2397 \backslash_{1.240}$ & $1.3988 \backslash_{1.399}$ & & & & & & & & \\
\hline 2 & $1.6667 \backslash_{1.667}$ & $2.1105 \backslash_{2.110}$ & $2.1930 \backslash 2.193$ & & & & & & & \\
\hline 3 & $1.8637 \backslash_{1.864}$ & $2.4562 \backslash_{2.456}$ & $2.6948 \backslash 2.695$ & $2.7416 \backslash 2.742$ & & & & & & \\
\hline 4 & $1.8841 \backslash_{1.884}$ & $2.5275 \backslash 2.528$ & $2.8457 \backslash 2.846$ & $2.9887 \backslash 2.989$ & $3.0201 \backslash_{3.020}$ & & & & & \\
\hline 5 & $1.7972 \backslash 1.797$ & $2.4365 \backslash 2.436$ & $2.7834 \backslash 2.783$ & $2.9813 \backslash 2.981$ & $3.0855 \backslash_{3.085}$ & $3.1112 \backslash 3.111$ & & & & \\
\hline 6 & $1.6564 \backslash 1.656$ & $2.2634 \backslash 2.263$ & $2.6128 \backslash 2.613$ & $2.8365 \backslash 2.836$ & $2.9884 \backslash 2.988$ & $3.0796 \backslash 3.080$ & $3.1040 \backslash_{3.104}$ & & & \\
\hline 7 & $1.4958 \backslash_{1.496}$ & $2.0577 \backslash 2.058$ & $2.3959 \backslash 2.396$ & $2.6288 \backslash_{2.629}$ & $2.8072 \backslash 2.807$ & $2.9450 \backslash 2.945$ & $3.0348 \backslash_{3.035}$ & $3.0599 \backslash_{3.060}$ & & \\
\hline 8 & $1.3343 \backslash 1.334$ & $1.8467 \backslash_{1.847}$ & $2.1666 \mid 2.167$ & $2.3987 \backslash 2.399$ & $2.5897 \backslash 2.590$ & $2.7548 \backslash 2.755$ & $2.8918 \backslash 2.892$ & $2.9844 \mid 2.984$ & $3.0106 \backslash 3.011$ & \\
\hline 9 & $1.1815 \backslash_{1.181}$ & $1.6444 \backslash_{1.644}$ & $1.9424 \backslash 1.942$ & $2.1675 \backslash 2.167$ & $2.3617 \backslash 2.362$ & $2.5404 \backslash 2.540$ & $2.7048 \backslash_{2.705}$ & $2.8454 \backslash_{2.845}$ & $2.9416 \backslash 2.942$ & $2.9690 \backslash 2.969$ \\
\hline 10 & 1.0415 & 1.4571 & 1.7317 & 1.9458 & 2.1369 & 2.3197 & 2.4974 & 2.6652 & 2.8100 & 2.9095 \\
\hline
\end{tabular}

a. The exact values given by Albrecher et al. (2005) are in smaller size after $\backslash$.

Table 6. Approximations for the third moment $W_{b}^{(3)}(u)$.

\begin{tabular}{|c|c|c|c|c|c|c|c|c|c|c|c|}
\hline$b^{u}$ & 0 & 1 & 2 & 3 & 4 & 5 & 6 & 7 & 8 & 9 & 10 \\
\hline 0 & 3.5755 & & & & & & & & & & \\
\hline 1 & 8.4888 & 20.770 & & & & & & & & & \\
\hline 2 & 19.421 & 45.111 & 77.832 & & & & & & & & \\
\hline 3 & 26.504 & 61.464 & 104.56 & 160.74 & & & & & & & \\
\hline 4 & 26.994 & 62.598 & 106.45 & 163.03 & 239.93 & & & & & & \\
\hline 5 & 23.379 & 54.214 & 92.189 & 141.20 & 207.79 & 300.33 & & & & & \\
\hline 6 & 18.484 & 42.863 & 72.888 & 111.63 & 164.32 & 237.87 & 341.11 & & & & \\
\hline 7 & 13.877 & 32.179 & 54.720 & 83.809 & 123.36 & 178.62 & 256.75 & 366.92 & & & \\
\hline 8 & 10.113 & 23.453 & 39.881 & 61.081 & 89.909 & 130.19 & 187.17 & 268.21 & 382.71 & & \\
\hline 9 & 7.2458 & 16.803 & 28.573 & 43.762 & 64.415 & 93.273 & 134.11 & 192.22 & 275.06 & 392.21 & \\
\hline 10 & 5.1400 & 11.920 & 20.269 & 31.044 & 45.694 & 66.166 & 95.133 & 136.36 & 195.18 & 279.13 & 397.90 \\
\hline
\end{tabular}

used in an arbitrary Sparre Andersen model. The iteration and the recursion prove helpful in achieving the goal. I think that more extensive applications about these methods can be found. Obviously, the iteration method can also be used to compute the Gerber-Shiu penalty function in Sparre Andersen model, even more complicated model.

\section{References}

[1] B. De Finetti, "Su un'Impostazione Alternativa Della Teoria Collettiva del Rischio," Transactions of the XVth International Congress of Actuaries, Vol. 2, No. 1, 1957, pp. 433-443.

[2] X. S. Lin, G. E. Willmot and S. Drekic, "The Classical Risk Models with a Constant Dividend Barrier: Analysis of the GERBER-Shiu Discounted Penalty Function," In- surance: Mathematics and Economics, Vol. 33, No. 3, 2003, pp. 551-566.

[3] D. C. M. Dickson and H. R. Waters, "Some Optimal Dividend Problems," Astin Bulletin, Vol. 34, No. 1, 2004, pp. 49-74. doi:10.2143/AST.34.1.504954

[4] H. U. Gerber, E. S. W. Shiu and N. Smith, "Methods for Estimating the Optimal Dividend Barrier and the Probability of Ruin," Insurance: Mathematics and Economics, Vol. 42, No. 2, 2008, pp. 243-254. doi:10.1016/j.insmatheco.2007.02.002

[5] E. S. Anderson, "On the Collective Theory of Risk in Case of Contagion between Claims," Bulletin of the Institute of Mathematics and Its Applications, Vol. 12, No. 2, 1957, pp. 275-279.

[6] S. Li and J. Garrido, "On a Class of Renewal Risk Models with a Constant Dividend Barrier," Insurance: Mathematics and Economics, Vol. 35, No. 3, 2004, pp. 691-701. 
doi:10.1016/j.insmatheco.2004.08.004

[7] M. M. Claramunt, M. Marmol and R. Lacayo, "On the Probability of Reaching a Barrier in an Erlang(2) Risk Process," Working Paper No. 24, Universitat Autonoma de Barcelona, Cerdanyola del Vallès, 2004.

[8] H. Albrecher, M. Mercgravee Claramunt and M. Marmol, "On the Distribution of Dividend Payments in a Sparre
Andersen Model with Generalized Erlang(n) Interclaim Times," Insurance: Mathematics and Economics, Vol. 37, No. 2, 2005, pp.324-334. doi:10.1016/j.insmatheco.2005.05.004

[9] Erwin Kreyszig, "Introductory Functional Analysis with Applications," John Wiley \& Sons, Hoboken, 1978, pp. 299-302. 